\title{
Peritoneal Tuberculosis: Diagnostic Options
}

\author{
N. Lal ${ }^{1}$ and V. Soto-Wright ${ }^{1,2 *}$ \\ ${ }^{1}$ Department of Obstetrics and Gynecology, Boston Medical Center, Boston, MA \\ ${ }^{2}$ Boston University School of Medicine, Boston, MA
}

\begin{abstract}
Background: Extrapulmonary tuberculosis has vague symptoms and few signs. It is essential to recognize and diagnose this curable disease prior to performing definitive surgery. Newer tests such as DNA or RNA amplification allow for early diagnosis but have limitations.

Case: We report a case of peritoneal tuberculosis in an immigrant woman. She had vague symptoms of low-grade fever, mild abdominal pain, obstipation, and bloating. Diagnostic laparoscopy was performed to establish the diagnosis. Tuberculosis was confirmed by DNA extraction from the frozen section specimen with subsequent analysis using polymerase chain reaction.

Conclusion: Peritoneal tuberculosis is a disease that often simulates malignancies. With the increasing prevalence of human immunodeficiency virus in developed countries, tuberculosis is also on the rise and should be considered in the differential diagnosis of a patient with an abdominal/ pelvic mass and ascites. Infect. Dis. Obstet. Gynecol. 7:244-247, 1999. (O) 1999 Wiley-Liss, Inc.
\end{abstract}

$T_{\mathrm{s}}^{\mathrm{h}}$ he incidence of tuberculosis in the United States has recently increased due to the resurgence of human immunodeficiency virus. ${ }^{1}$ Individuals at high risk for developing tuberculosis include patients with acquired immunodeficiency syndrome, immigrants from underdeveloped countries, the urban poor, prisoners, and nursing home residents. Nonspecific symptoms and signs are a hallmark of tuberculosis, necessitating a high index of suspicion to diagnose this disease. ${ }^{2}$

\section{CASE}

A 33-year-old woman, gravida 2, para 1, who immigrated from Cameroon to the United States in August 1998 presented to the urgent care department reporting bloating, obstipation, vague abdominal pain, and low-grade fever of nine months' duration. She had a normal spontaneous vaginal delivery in December 1997. Physical examination was remarkable for a distended abdomen with an ascitic fluid wave but no tenderness or mass. The pelvic exam revealed a friable cervical ectropion but no cervical motion tenderness. Her uterus was anteverted, nontender, and normal sized. Both ovaries were nontender and palpable in the posterior cul de sac.

Laboratory investigations showed a hemoglobin level of $9.2 \mathrm{~g} / \mathrm{dL}$ with hypochromic, normocytic red blood cell indices, platelets of $487 \mathrm{~K} / \mathrm{UL}$ and an erythrocyte sedimentation rate (ESR) of $49 \mathrm{~mm} / \mathrm{h}$. Liver and kidney function tests were normal except for the presence of hypoalbuminemia (albu$\min , 2.5 \mathrm{~g} / \mathrm{dL}$ ). A chest X-ray was normal. Her purified protein derivative level was positive, measuring $10 \mathrm{~mm}$. A Papanicolaou test showed benign cellular changes. An abdominal pelvic ultrasound revealed loculated ascites with a normal uterus measuring $6.8 \mathrm{~cm}$ and bilateral ovarian enlargement with complex ovarian cysts. A computed tomography scan of the abdomen and pelvis was consistent with the ultrasound results.

*Correspondence to: Valena Soto-Wright, MD, Assistant Professor, Boston University School of Medicine, 1 Boston Medical Center Place, Boston, MA 02115. E-mail: valena.sotowright@bmc.org 

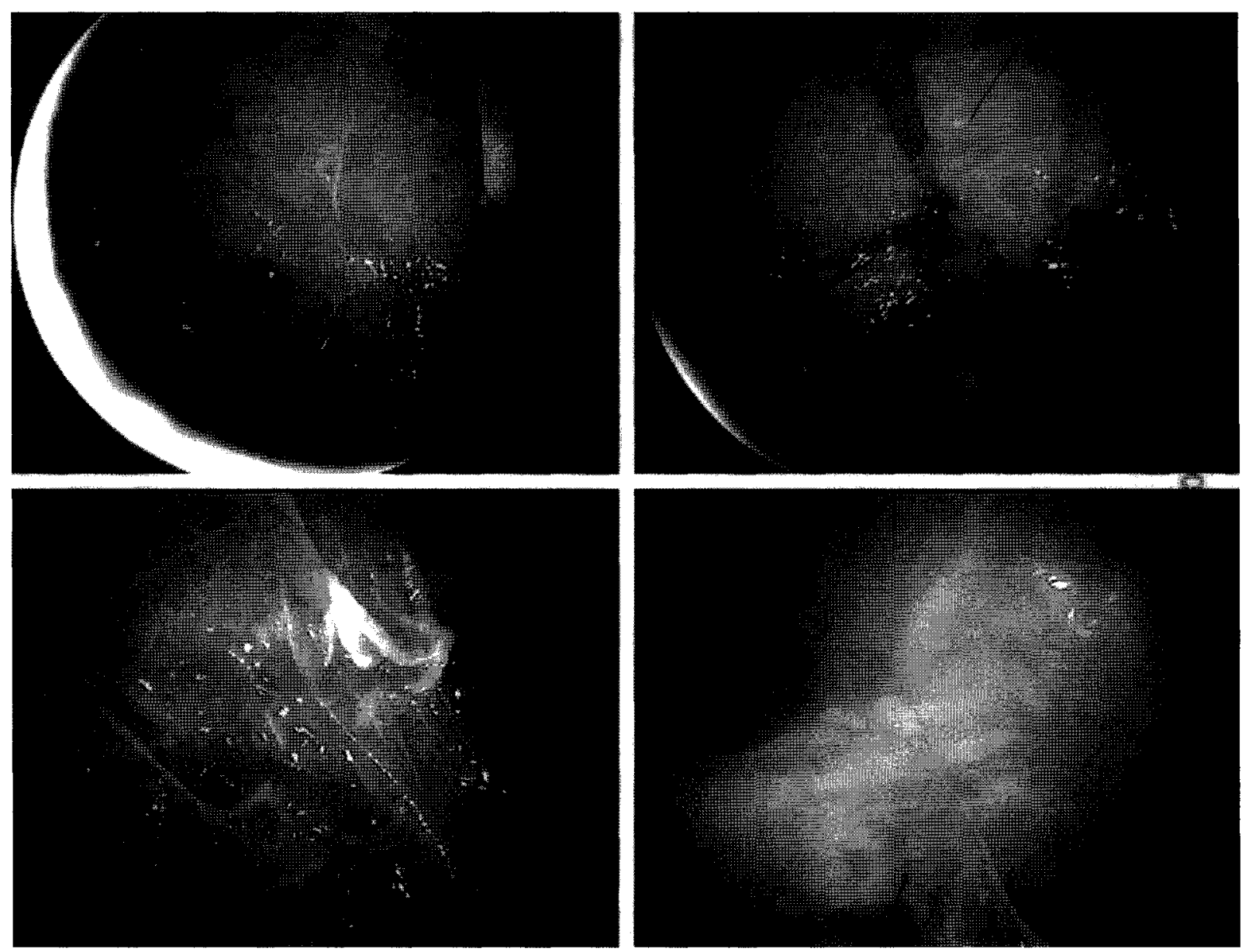

Fig. I. Laparoscopic view of left adnexa with tuberculous peritoneal adhesions and loculated ascites.

Diagnostic paracentesis revealed an exudative ascitic fluid with predominant lymphocytosis. The chemistries on the ascitic fluid showed lactate dehydrogenase of $934 \mathrm{U} / \mathrm{L}$; albumin, $2.3 \mathrm{~g} / \mathrm{dL}$; glucose, $11 \mathrm{mmol} / \mathrm{L}$; lipase, $73 \mathrm{~g} / \mathrm{dL}$; and total protein, $6.7 \mathrm{~g} / \mathrm{dL}$. Gram staining of the fluid showed $0-5$ polymorphonuclear cells and no microorganisms. Ascitic fluid was sent for aerobic, anaerobic, and mycobacterial culture. The patient was then referred to the Division of Gynecologic Oncology to rule out the diagnosis of ovarian carcinoma. The patient's preoperative CA 125 level was elevated to $138 \mathrm{U} / \mathrm{mL}$. Open diagnostic laparoscopy was performed and showed several thick, caseous peritoneal adhesions with multiple peritoneal nodules and plaques covering the abdominopelvic peritoneum. The ascites was loculated (Fig 1 ) in both the upper abdomen as well as the pelvis. Uterus and adnexa were encased in thick caseous adhesions limiting the view of the adnexa. Evaluation of a frozen section of the peritoneal nodule showed granuloma formation with caseating necrosis. 
Analysis by polymerase chain reaction (PCR) of the specimen demonstrated a DNA sequence diagnostic of Mycobacterium tuberculosis. The patient was started on isoniazid, rifampicin, and ethambutol, the standard triple drug regimen for extrapulmonary tuberculosis. ${ }^{3}$

The patient's 4-week postoperative follow-up evaluation showed a decrease in the CA 125 level to $40 \mathrm{U} / \mathrm{mL}$. Postoperative pelvic ultrasound showed resolution of ascites and normal ovaries with no evidence of any residual cystic change.

\section{DISCUSSION}

Tuberculous peritonitis usually follows either the direct spread of tubercle bacilli from ruptured lymph nodes, intraabdominal/pelvic organs, or hematologic seeding from a primary focus elsewhere in the body. The differential diagnosis in a patient with ascites and pelvic mass includes ovarian malignancies, cirrhosis with portal hypertension, Crohn disease, gastrointestinal malignancies, malignant lymphomas, and sarcoidosis.

Infertility and irregular menses are the most common symptoms of genital tuberculosis. ${ }^{4}$ Normal menstruation and a recent normal vaginal delivery in our patient did not suggest the presence of genital tuberculosis. Her chest X-ray was also normal but $50 \%$ of patients with active tuberculous peritonitis have negative chest radiographs. Nonspecific signs of tuberculous peritonitis include anemia of chronic disease, elevated ESR, ascitic lactate dehydrogenase greater than $90 \mathrm{U} / \mathrm{L},{ }^{5}$ ascitic lymphocytosis, complex ascites, ${ }^{6}$ and elevated levels of CA $125 .{ }^{7}$ The presence of these nonspecific signs (positive purified protein derivative skin test, elevated ESR, ascitic fluid lactate dehydrogenase level of $934 \mathrm{U} / \mathrm{L}$, lymphocytic ascitic fluid, and complex ascites) favored the diagnosis of peritoneal tuberculosis prior to diagnostic laparoscopy.

Mycobacterial culture is the gold standard for diagnosing tuberculosis despite its long incubation period and requirement for biochemical testing to identify the isolated microorganism. ${ }^{8}$ Polymerasechain-reaction-based assay proved to be a valuable diagnostic tool for Mycobacterium tuberculosis. The direct amplification of a defined DNA sequence of Mycobacterium tuberculosis established the diagnosis within 48 hours. This is in contrast to the 8 weeks required for mycobacterial culture. Testing by PCR can be performed on sputum samples, body fluids, and tissue specimens of patients suspected of having pulmonary or extrapulmonary tuberculosis. ${ }^{8,9}$ However, in cases of extrapulmonary tuberculosis, the sensitivity of PCR testing may be greatly reduced, as the bacterial load in the peritoneal, pleural, and joint cavities is very low. Inhibitors to PCR may also be found in extrapulmonary specimens, limiting its usefulness in extrapulmonary tuberculosis. Despite these limitations, PCR amplification can be useful for the detection of mycobacterial DNA in frozen section specimens, allowing a specific diagnosis to be made rapidly. ${ }^{8-10}$

Prior to performing a more invasive, definitive surgical procedure with increased morbidity, open diagnostic laparoscopy should be considered to establish a diagnosis of tuberculosis. ${ }^{11,12}$ Open laparoscopy is imperative, as small bowel loops may be adherent to the anterior peritoneum secondary to the peritonitis. Additionally, aspiration of ascites prior to insufflation prevents air bubbles from obscuring the laparoscopic view. This enables the clinician to establish a clinical diagnosis, obtain a specimen for frozen section, and rule out coexisting disease while preserving the fertility of the patient.

\section{REFERENCES}

1. Snider DE Jr, Roper WL. The new tuberculosis. N Engl J Med 1992;326:703-705.

2. Groutz A, Carmon E, Gat A. Peritoneal tuberculosis versus advanced ovarian cancer: a diagnostic dilemma. Obstet Gynecol 1998;91:868.

3. Bass JB Jr, Farer LS, Hopewell PC, et al. Treatment of tuberculosis and tuberculosis infection in adults and children. Am J Respir Crit Care Med 1994;149:13591374.

4. Figueroa-Damian R, Martinez-Velazco I, VillagranaZesati R, Arredondo-Garcia JL. Tuberculosis of the female reproductive tract: effect on function. Int J Fertil Menopausal Stud 1996;41:430-436.

5. Shakil AO, Korula J, Kanel GC, Murray NG, Reynolds TB. Diagnostic features of tuberculous peritonitis in the absence and presence of chronic liver disease: a case control study. Am J Med 1996;100:179-185.

6. Yapar EG, Ekici E, Karasahin E, Gokmen O. Sonographic features of tuberculous peritonitis with female genital tract tuberculosis. Ultrasound Obstet Gynecol 1995;6:121-125.

7. O'Riordan DK, Deery A, Dorman A, Epstein OE. Increased CA 125 in a patient with tuberculous peritonitis: case report and review of published works. Gut 1995; $36: 303-305$. 
8. Aslanzadeh J, de la Viuda M, Fille M, Smith WB, Namdari $\mathrm{H}$. Comparison of culture and acid-fast bacilli stain to PCR for detection of Mycobacterium tuberculosis in clinical samples. Mol Cell Probes 1998;12:207-211.

9. Salian NV, Rish JA, Eisenach KD, Gave MD, Bates JH. Polymerase chain reaction to detect Mycobacterium tuberculosis in histologic specimens. Am J Respir Crit Care Med 1998;158:1150-1155.
10. Chang GM, Yuen YK, Chan KS, et al. Single-tube nested PCR in the diagnosis of tuberculosis. J Clin Pathol 1996;49:290-294.

11. McLaughlin S, Jones T, Pitcher M, Evans P. Laparoscopic diagnosis of abdominal tuberculosis. Aust NZ J Surg 1998;68:599-601.

12. Hossain J, Kinirons M, Lewis RR. Laparoscopy does have a role in tuberculous peritonitis. BMJ 1996;313: $1145-1146$ 


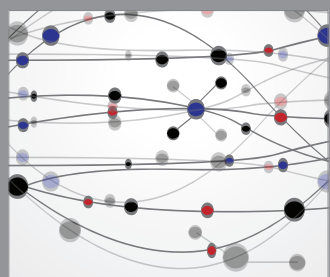

The Scientific World Journal
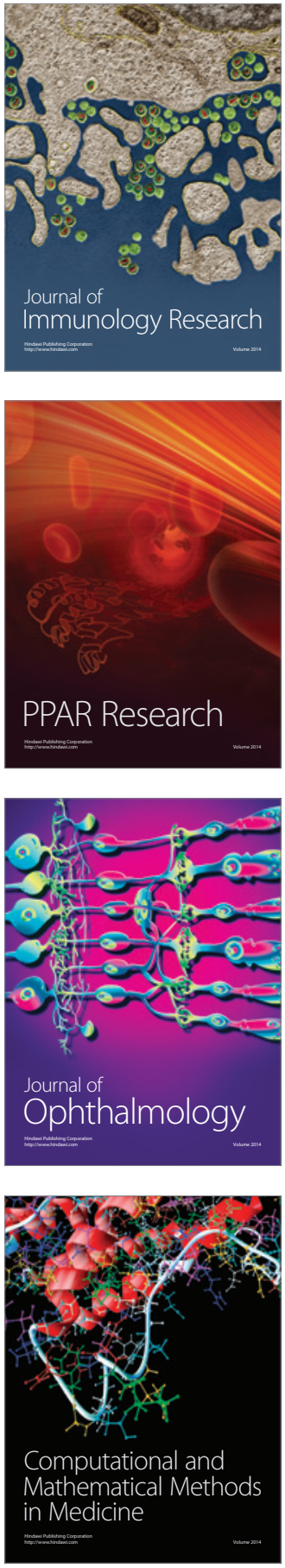

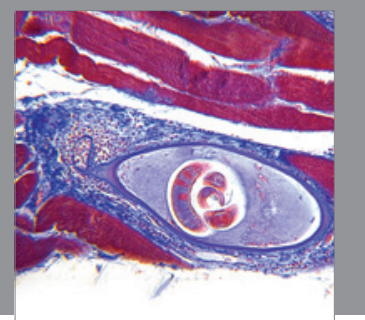

Gastroenterology

Research and Practice
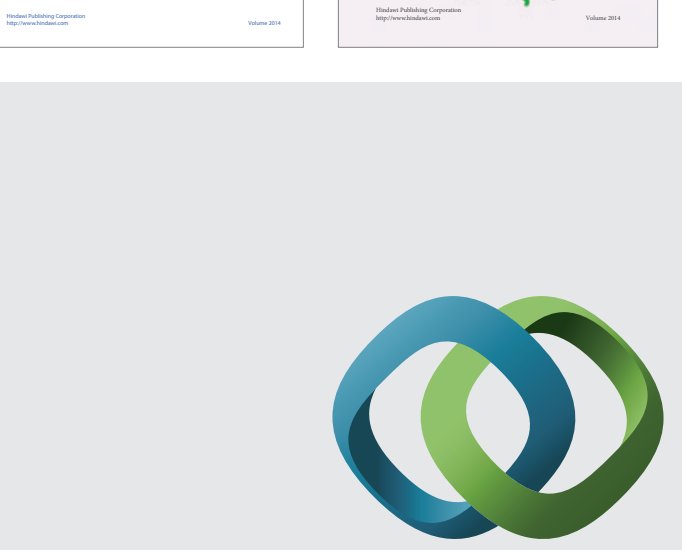

\section{Hindawi}

Submit your manuscripts at

http://www.hindawi.com
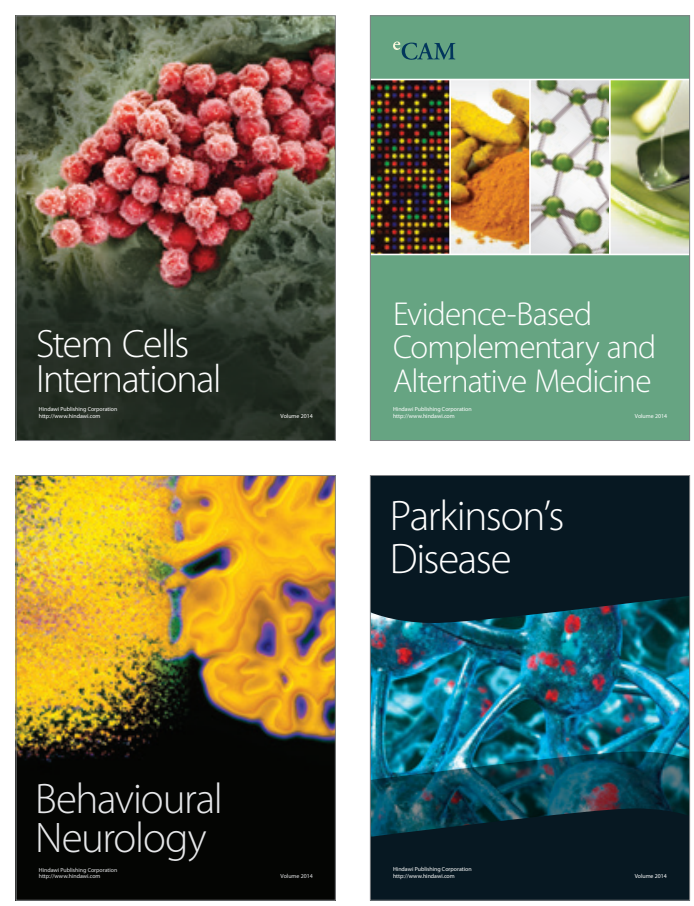

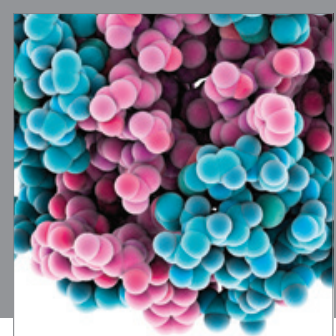

Journal of
Diabetes Research

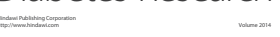

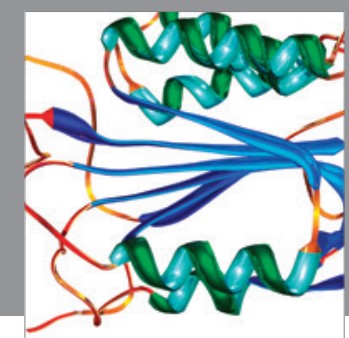

Disease Markers
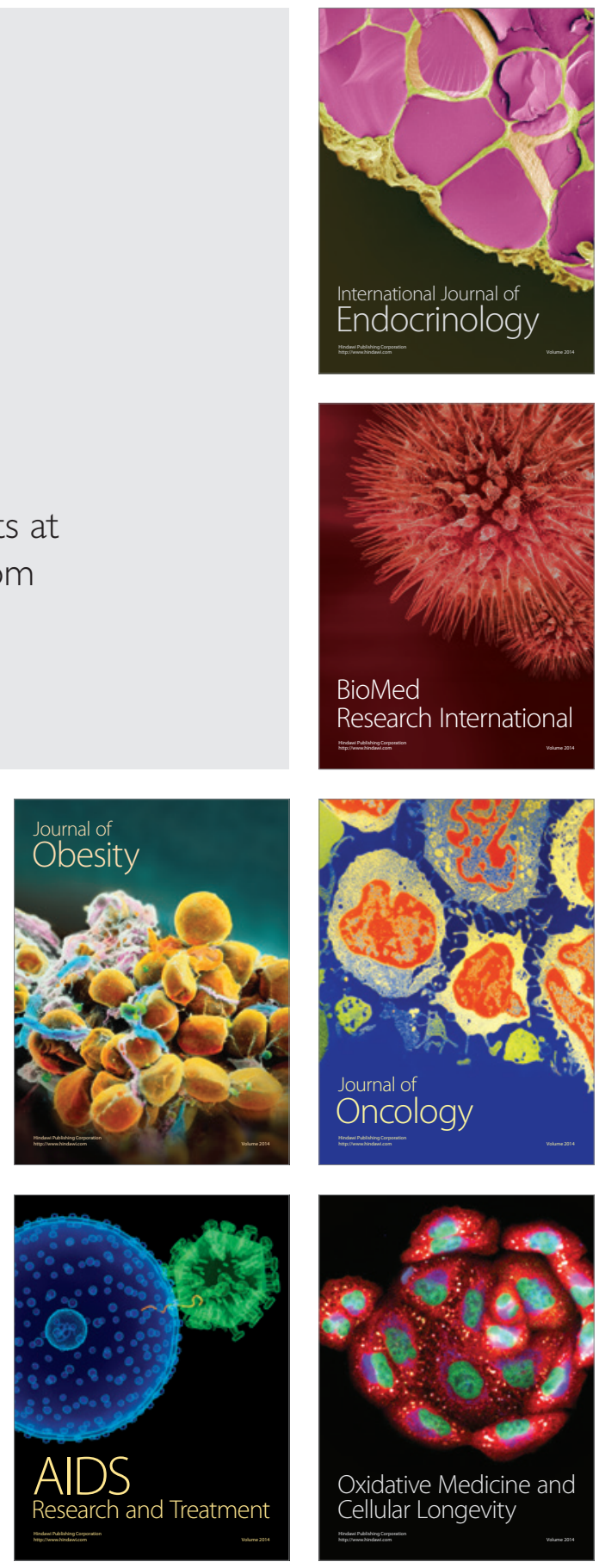